\title{
Francine Saillant
}

Anthropologue, École des sciences infirmières

et Centre de recherche sur les services communautaires Université Laval.

(1985)

\section{"Les discours sur le cancer en contexte clinique moderne: le sens de la mort en question"}

Un document produit en version numérique par Jean-Marie Tremblay, bénévole, professeur de sociologie au Cégep de Chicoutimi

Courriel: jean-marie tremblay@uqac.ca

Site web pédagogique : http://www.uqac.ca/jmt-sociologue/

Dans le cadre de la collection: "Les classiques des sciences sociales"

Site web: http://www.uqac.ca/Classiques des sciences sociales/

Une collection développée en collaboration avec la Bibliothèque Paul-Émile-Boulet de l'Université du Québec à Chicoutimi

Site web: http://bibliotheque.uqac.ca/ 
Cette édition électronique a été réalisée par Jean-Marie Tremblay, bénévole, professeur de sociologie au Cégep de Chicoutimi à partir de l'article de :

Francine Saillant, "Les discours sur le cancer en contexte clinique moderne : le sens de la mort en question".

Un article publié dans l'ouvrage sous la direction de Raymond Lemieux et Réginal Richard, Survivre... La religion et la mort, pp. 143-161. Les Cahiers de recherches en sciences de la religion, vol. 6, 1985. Montréal: Les Éditions Bellarmin, 1985, $285 \mathrm{pp}$.

Mme Saillant est anthropologue à École des sciences infirmières et chercheure au Centre de recherche sur les services communautaires Université Laval.

[Autorisation formelle accordée par l'auteure de diffuser tous ses travaux le 4 novembre 2005.]

\section{Courriel : francine.saillant@ant.ulaval.ca}

Polices de caractères utilisée :

Pour le texte: Times New Roman, 14 points.

Pour les citations : Times New Roman 12 points.

Pour les notes de bas de page : Times New Roman, 12 points.

Édition électronique réalisée avec le traitement de textes Microsoft Word 2004 pour Macintosh.

Mise en page sur papier format : LETTRE (US letter), 8.5” x 11”)

Édition numérique réalisée le 5 décembre 2006 à Chicoutimi, Ville de Saguenay, province de Québec, Canada. 


\section{Francine Saillant}

Mme Saillant est anthropologue à École des sciences infirmières et chercheure au Centre de recherche sur les services communautaires Université Laval.

\section{"Les discours sur le cancer en contexte clinique moderne : le sens de la mort en question".}

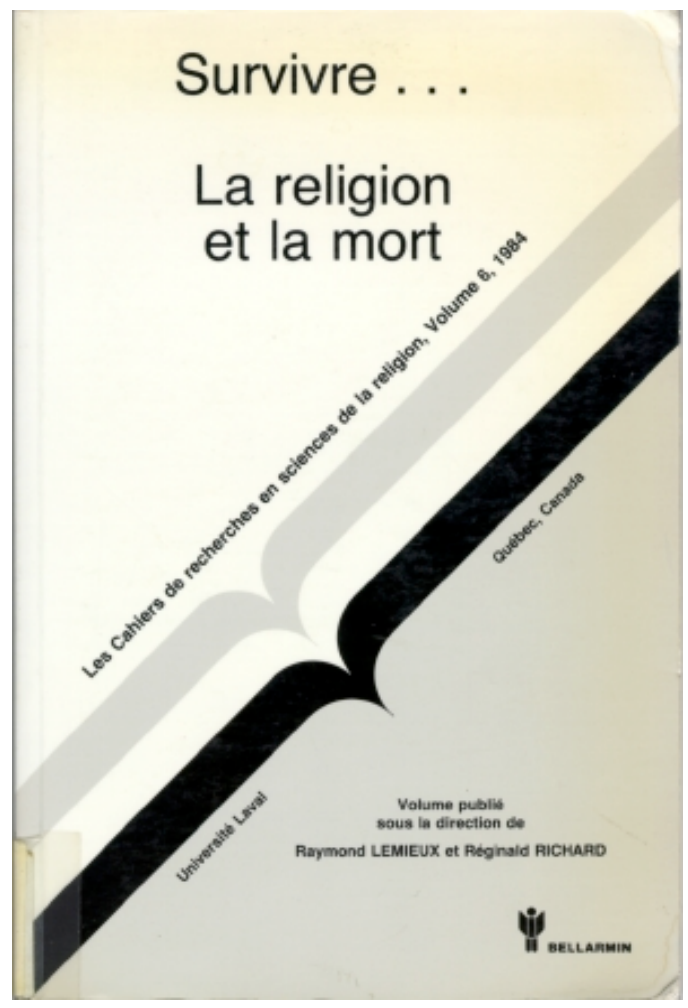

Un article publié dans l'ouvrage sous la direction de Raymond Lemieux et Réginal Richard, Survivre... La religion et la mort, pp. 143-161. Les Cahiers de recherches en sciences de la religion, vol. 6, 1985. Montréal: Les Éditions Bellarmin, 1985, $285 \mathrm{pp}$. 


\section{Table des matières}

$\underline{\text { Introduction }}$

1. Du déni de la mort à la mort d'un monde

1.1. L'institution garante du refoulement

1.2. Mourir hier et aujourd'hui

1.3. La mort à la mode

1.4. Le discours sur la mort comme métaphore

2. Le cancer comme production discursive

2.1. Une résidence pour personnes cancéreuses

2.2. Le cancer en tant que phénomène social

3. Le discours sur le cancer

3.1. Le discours des soignants

3.2. Le discours des soignés

$\underline{\text { Conclusion }}$

$\underline{\text { Références }}$ 
Francine Saillant

Mme Saillant est anthropologue à École des sciences infirmières et chercheure au

Centre de re-cherche sur les services communautaires Université Laval.

"Les discours sur le cancer en contexte clinique moderne:

le sens de la mort en question".

Un article publié dans l'ouvrage sous la direction de Raymond Lemieux et Réginal Richard, Survivre... La religion et la mort, pp. 143-161. Les Cahiers de recherches en sciences de la religion, vol. 6, 1985. Montréal: Les Éditions Bellarmin, 1985, $285 \mathrm{pp}$.

\section{Introduction}

Retour à la table des matières

Le présent essai a pour but de signaler certains résultats d'une recherche doctorale en cours concernant l'ethnographie d'un établissement québécois francophone réservé à une population de personnes cancéreuses pour la plupart en phase curative de leur traitement ${ }^{1}$. Les discours populaires et savants sur le cancer seront ici particulièrement discutés et contrastés.

L'objet de cette thèse ne consiste pas en l'analyse du discours sur la mort en milieu clinique. Au début de cette recherche, nous avions d'ailleurs postulé que pour entendre le discours sur le cancer dans sa globalité, il fallait justement éviter provisoirement de se concentrer sur la question du devenir de la personne cancéreuse, c'est-à-dire de sa

1 Il s'agit d'une thèse en anthropologie médicale portant plus spécifiquement sur les productions discursives reliées au cancer. Le travail final sera présenté au département d'anthropologie de l'Université McGill (Montréal). La cueillette des données a débuté en mars 1982 et sera achevée à la fin de l'été 1983. Notre présentation a ainsi pour statut celui d'une note de recherche : on ne saurait donc attribuer à ces hypothèses les qualités de résultats définitifs. 
mort probablement prochaine : cette position, jugions-nous, allait nous permettre de ne pas stigmatiser ces personnes, en leur conférant, comme c'est très souvent l'habitude, un statut de demi-vivant ${ }^{2}$.

Nous avons déjà émis l'hypothèse que le cancer représente, dans la société québécoise, et probablement dans les pays occidentaux industrialisés où on retrouve une forte morbidité attribuable au cancer, un symbole de mort (Saillant, 1982). Les représentations de la maladie dans les cultures dites savantes (médicales, techno-scientifique) et populaires (les soignés des classes moyennes et ouvrières) avaient été comparées. Il nous semblait alors que pour l'un et l'autre des deux milieux, le symbole agissait également comme un symbole de mort. Cette hypothèse sera ici discutée à nouveau : on tentera cependant de montrer que le discours sur le cancer agit véritablement dans la culture savante comme un discours sur la mort, en tant qu'objet d'échec, alors que dans la culture populaire, il s'agit peut-être d'un discours sur la mort, mais plus vraisemblablement d'un discours sur la modernité porteuse de mort.

Dans un premier temps, la littérature récente sur la mort sera discutée. On s'intéressera particulièrement à la proposition suivante : le discours de certains chercheurs ayant analysé la soi-disant évacuation de la mort dans les formations sociales euro-américaines et industrialisées peut-il être entendu comme un discours ayant d'abord pour objet la mort d'un monde?

Dans un deuxième temps, le lieu d'insertion et les conditions de production de cette ethnographie seront présentés.

Nous décrirons, dans un troisième temps, le discours des soignants et celui des soignés, après avoir postulé que le cancer, s'il est la deuxième cause de mortalité au Québec, doit être aussi regardé comme un phénomène social de premier plan nous permettant d'atteindre le discours de notre société sur ses propres limites, que cellesci soient d'ordre biologique, écologique, économique ou symbolique.

2 On peut lire une position récente sur cette question dans un numéro de Santé mentale au Québec (Saillant 1973 : 158-160). 


\title{
1. Du déni de la mort à la mort d'un monde
}

\author{
1.1. L'institution garante du refoulement
}

Retour à la table des matières

Le thème du déni de la mort en Occident judéo-chrétien domine la littérature sur la mort au moins depuis la publication des travaux de Kübler-Ross, en 1970. On death and dying (1970) a introduit une coupure dans la réflexion des scientifiques et des professionnels, concernant le processus du mourir, les étapes d'acceptation de la finalité du devenir humain ${ }^{3}$ et les stratégies d'évacuation en oeuvre par les soignants et l'institution hospitalière pour effacer ou déplacer la parole de ceux que l'on a identifiés comme des patients en phase terminale, c'est-à-dire ceux pour qui tout espoir de recouvrement de la santé, à l'aide des moyens connus du système bio-médical, est jugé impossible.

Cette réflexion a ouvert la voie à cette « nouvelle sensibilité thérapeutique $»{ }^{4}$ face au vécu de cette phase terminale, dont le death and dying movement constitue à cet égard le meilleur exemple ${ }^{5}$. Au Qué-

3 Ces stades étant décrits successivement comme : le déni et l'isolement; l'agressivité, la révolte ; la recherche des bénéfices secondaires ; la dépression ; l'acceptation.

4 Cette nouvelle sensibilité thérapeutique déborde d'ailleurs du champ du mourir, puisqu'elle a eu emprise sur différents domaines. Comme l'a remarqué Lasch (1979), les années '70 ont été l'âge de prédilection des nouvelles thérapies de toutes sortes, alors qu'une partie de la jeunesse a délaissé l'action sociale et les préoccupations politiques au profit de valeurs promouvant le bienêtre personnel, le développement du moi, etc. C'est donc aussi dans la mêlée de cette nouvelle culture que s'est inscrite cette vague de reprise en charge des mourants par la communauté.

5 Pour une revue de ce mouvement, cf. Levinson 1979. 
bec, ce mouvement se cristallise autour d'expériences nouvelles d'approches au mourant, telles que l'accompagnement à la maison ou à l'hôpital et, bientôt, un mouroir hors des murs hospitaliers (Maison Michel-Sarrazin, à Québec).

Différentes études, dont celle de Glazer et Strauss (1965), ont en effet montré que l'institution hospitalière, en concentrant à son maximum les expériences du mourir sous son toit, a permis le refoulement de la question de la mort, laissant à la maladie (l'objet même du discours médical) le centre de la convergence des pratiques professionnelles, en exerçant de surcroît un contrôle de par le silence imposé autour de l'échec des actes thérapeutiques ${ }^{6}$.

Herzlich (1976), reprenant Gorer (1955), a ainsi noté que le tabou de mort aurait remplacé ce que fut le tabou du sexe et, à la suite d'Ariès, a mentionné « qu'une fusion s'est opéré dans les représentations collectives entre l'angoisse de la mort et la peur de la maladie (Herzlich 1976 : 198). Certaines maladies prendraient par le fait même figure de symbole et cristalliseraient ainsi cette angoisse ; le cancer en serait l'exemple probablement le plus signifiant. Elle affirme ainsi que la mort serait indissociable de l'appareil des professions biomédicales, alors que la conscience de la mort demeurerait à l'ombre de l'espérance de guérison.

Toujours selon cet auteur, la figure de la mort aurait aujourd'hui échappé à cette idée biblique de malédiction collective, telle que le fléau, l'épidémie, pour n'être devenue qu'un événement à caractère individuel. Il n'est toutefois pas certain que cette affirmation soit des plus juste, puisque, si on accepte que certaines maladies rassemblent et condensent dans le monde contemporain les signes de la mort, il

6 Glazer et Strauss (1967) ont ainsi souligné que cette occultation vise d'ailleurs plus à protéger l'institution de la crise que représente la prise en considération de l'expression des mourants et de son entourage (perturbations émotives, brisure dans la continuité de la routine, scènes de désespoir, etc.), plutôt qu'à protéger le mourant et son entourage de l'angoisse. Comme Herzlich l'énonce : «Surtout, tout se passe comme si l'institution tout entière voulait avant tout réduire les conséquences déstructurantes d'un débordement émotionnel pour le travail quotidien » (p. 202). Pour Glazer et Strauss, le malade perd inévitablement au jeu de la feinte commune (Mutual pretense). 
faudrait donc aller puiser directement dans le discours sur ces dernières, afin de vérifier si vraiment ces idées de malédiction ont disparu. On se rendrait alors compte que, par exemple, les discours sur le cancer ou sur le " désastre écologique-cause-de-la-maladie » reprennent amplement la métaphore de la malédiction, qui, plutôt que d'être « décidée » par les dieux, serait aujourd'hui conséquence de la négligence des humains.

\subsection{Mourir hier et aujourd'hui}

\section{$\underline{\text { Retour à la table des matières }}$}

La préoccupation nouvelle face au phénomène du mourir et la critique de la médicalisation de cette expérience largement repoussée vers les institutions ont fait naître, comme on l'a affirmé, une multitude d'expériences nouvelles nées, entre autres, dans la foulée du mouvement de prise en charge communautaire de la santé. Des programmes ont été mis sur pied par et pour les professionnels, afin de développer une approche de rechange à l'égard des mourants, dans le but de réapprendre à vivre près d'eux.

De fait, « la mort à l'occidentale » serait largement opposée à la mort telle que la vivent les sociétés primitives et paysannes; différents travaux ont souligné cette coupure observée tant par les historiens que par les anthropologues ${ }^{7}$. Les sociétés occidentales, en multipliant leurs moyens de contrôle sur la nature, tendraient à exclure la mort comme elles cherchent à se séparer de la nature. La position de Thomas (1976: 99-100) résume fort bien cette tendance ${ }^{8}$ :

C'est ainsi qu'une société qualifiée de « traditionnelle » ou " d'archaïque », les deux termes sont aussi mauvais l'un que l'autre malgré certaines

7 Voir R. Jaulin 1967 ; Thomas 1976 ; Ziegler 1975 ; Hertz 1970.

8 Thomas, dans un ouvrage plus récent, a par ailleurs nuancé sa position, en se distanciant quelque peu de celle qu'il adoptait dans son Anthropologie de la mort. L'introduction de Mort et pouvoir laisse soupçonner une approche critique d'une opposition trop stricte entre la mort traditionnelle et la mort moderne. 
erreurs et déviations (notamment en périodes de crises, de psychoses collectives en relation avec les catastrophes naturelles), respecte la vie de l'homme et du monde (animal et cosmique), de l'homme-qui-ne-fait-qu'unavec-le-monde. Mais elle a besoin pour cela de s'appuyer sur le symbolique. Au contraire, une société à idéologie productiviste objective l'homme et détruit la nature, dans un même mouvement d'expansionnisme technologique à fin de rentabilité : l'homme devient alors " super-objet » de la société d'échange - l'expression de Herbert Marcuse - tandis que la nature subit la violence de l'exploitation par la pollution. Désormais le symbolique se réduit à l'imaginaire de l'idéologie-masque ou de discours publicitaire trompeur...

Matarasso (1977) révèle, par ailleurs, que la crise du Thanatos est triple : la mort classique étant disparue, nous serions passés à la mort technologique. Cette triple crise serait induite par le questionnement sur la matérialité des faits de la mort (sa définition), par la décomposition des rites de deuil dans les classes urbaines et par le pouvoir nouveau des classes technocratiques et professionnelles (Le., l'ensemble des professions d'orientations bio-médicales). Matarasso parle ainsi de renversement symbolique, en expliquant la place actuelle de la mort dans les formations sociales des sociétés complexes. Autant Ziegler (1975), Thomas (1976) que Hertz (1970) décrivent les mêmes oppositions structurales entre le système de la mort traditionnelle et le système de la mort occidentale. À une mort pauvre, fragmentée, en rupture avec les vivants, destructrice de l'ordre vivant, privée, cachée et réprouvée s'oppose une mort féconde, totalisante, unie aux vivants, publique, exposée et approuvée, ayant pouvoir de recycler l'ordre biosocial.

Ainsi, ce mouvement de réinsertion du mourant et de réhabilitation de son expérience dans la vie vise, entre autres, à« humaniser » l'expérience de la mort et de la maladie « terminale », mais au-delà, il s'inscrit comme un mouvement critique face aux effets néfastes des abus d'une société technocratique et professionnaliste, sur tous les cycles de la vie, y compris nécessairement le mourir. La situation de la mort dans les sociétés modernes dénoterait une rupture fondamentale, quant à la place occupée par l'humain dans le système social, alors réduit à sa simple fonctionnalité marchande : plus que le produit, ou l'effet de cette aliénation, cette situation en serait l'ultime représentant : 
En faisant silence sur l'événement thanatique, en privant l'homme du choix de l'instant de sa mort, en masquant son agonie et en refusant son statut au mourant, le système capitaliste détruit l'homme dans son être. Il le prive de l'expérience réflexive de la phase la plus importante de sa vie et l'empêche de prendre, à son terme, la mesure de son existence. Mais il y a plus : en vidant la mort de tout son sens existentiel qu'elle véhicule, le système capitaliste prive l'existence humaine de sa liberté, de sa signification eschatologique, donc de sa qualité de destin. L'homme est occulté à luimême (Ziegler 1975 : 299).

\subsection{La mort à la mode}

$\underline{\text { Retour à la table des matières }}$

Cette très abondante littérature ayant critiqué et analysé la place et le statut de la mort dans les sociétés traditionnelles en comparaison avec les sociétés modernes traduit toutefois une attitude quelque peu rousseauiste : on la retrouve dans le discours de presque tous les chercheurs, particulièrement ceux qui ont approché le phénomène sous ses angles socio-historico-anthropologiques. C'est, du moins, ce qu'avance Chamboredon (1976) dans son analyse de la mode scientifique qu'est cette effervescence des chercheurs autour de la mort, depuis 1970. Il interroge ainsi ce point de convergence théorique entre des auteurs comme Jaulin, Ariès ou Baudrillard (1976), qui est de voir dans l'attitude actuelle face à la mort la fin d'un rapport social, voir « le point extrême de la fin de tout » (p. 79).

À bon escient, Chamboredon compare ce discours autour de la mort au discours alarmiste des mass-média ; ce thème devient l'occasion d'exprimer des préoccupations et des inquiétudes sociales, telles que le pessimisme idéologique, l'anticapitalisme, le ressentiment, l'éli- 
tisme, l'anti-modernisme 9. L'histoire semble alors conçue comme une dégradation, tandis que la fascination pour la mort traduirait tout simplement une nostalgie traditionnaliste représentée par l'image idéalisée d'un affrontement lucide de la mort dans le passé.

\subsection{Le discours sur la mort comme métaphore}

$\underline{\text { Retour à la table des matières }}$

Les commentaires précédents n'appellent-ils pas à suggérer que tout discours sur la mort est aussi un discours sur la société et sur ses limites ? Le discours moderne sur la mort met en évidence que le monde capitaliste, s'il cherche à tout prix à se représenter comme une obligation au bonheur, à la joie de vivre (et à la reproductivité !), est aussi perçu par certains comme producteur de sa propre mort, en tant que première " civilisation » possédant les moyens de s'auto-détruire.

Thomas consacre d'ailleurs tout un chapitre de son livre à la mort des faits sociaux (fin des institutions, de certaines sociétés, etc...). Les faits sociaux sont dotés de temporalité ; la hantise de l'Apocalypse, le millénarisme prennent la forme de discours sur la mort des faits sociaux. Ne pourrait-on pas ajouter que certains discours analytiques et scientifiques sur la mort (tels que ceux de Ziegler et de Baudrillard),

9 Voici l'hypothèse : « Le tabou dont la société capitaliste marchande frappe la mort n'est qu'un aspect d'une stratégie d'occultation plus vaste : c'est la stratégie culturelle que la classe dominante met en oeuvre pour sauvegarder, masquer et renforcer le système d'inégalités qui la privilégie. L'ensemble des symboles qui gouvernent la société marchande est ainsi affecté par une sorte de surdétermination. La réintroduction de la mort dans le champ du discours ne rétablit donc pas, par elle-même, l'intégralité de l'homme. Pour que cet homme vérifié, mutilé, privé de la claire conscience de sa finitude, donc de sa liberté, puisse retrouver une existence destinale, il faudra que l'ancien champ de réalité soit détruit pierre par pierre ; la réification totale des existences humaines par la société marchande ne peut être détruite que par une totalité de significations alternatives » (Ziegler $1975: 14)$. 
seraient en réalité une métaphore d'un autre discours qui a d'abord et avant tout pour objet la mort d'un monde, et celle de l'Homme 10 ?

Le discours écologique pourrait à ce titre occuper une place similaire, en tant que discours sur la décadence d'un monde en pourriture, et en mutation.

Valade (1977), en cernant l'évolution de l'idée de décadence depuis l'Empire romain jusqu'à nos jours, abonde en exemples qui figurent jusqu'à quel point chaque société possède un discours sur ses limites et sur sa finitude, lequel « encombre et surcharge la conscience douloureuse du devenir temporel qui abolit les valeurs qu'on croyait éternelles » (Valade $1977:$ 76) ${ }^{11}$. L'idée de décadence, en ce sens, se rapproche nettement de l'idée d'une société mortifère, vouée au désastre écologique, mutilante, telle qu'elle est connotée, entre autres, par les socio-anthropologues et les historiens du mourir.

\title{
2. Le cancer comme production discursive
}

\author{
2.1. Une résidence pour personnes cancéreuses
}

$\underline{\text { Retour à la table des matières }}$

10 La thématique de la mort de l'Homme est présente, comme on le sait, chez des auteurs tels que Foucault ou Lévi-Strauss. Par ailleurs, la proposition selon laquelle les discours contemporains sur la mort pourraient être lus dans le sens d'un discours sur la mort de l'Homme comme production historique n'a pas encore été, en tant que telle, explorée. Cette proposition mériterait bien sûr d'être documentée davantage.

11 Par exemple, le problème de la mort de Rome s'est posé bien avant que Rome ne meure : « La hantise d'un épuisement du soi, d'un avilissement de moeurs, de l'absence des grands hommes est déjà manifeste chez Lucrèce, Cicéron et Salluste ». (Valade $1977:$ 76). 
Le lieu d'observation que nous avons privilégié est une hôtellerie hospitalière d'une capacité de 99 lits destinée plus spécifiquement à des personnes cancéreuses. Ce pavillon est occupé à 95,95\% et reçoit environ 1000 patients par année. L'âge moyen de la population est de 57 ans ; $60 \%$ de la clientèle est de sexe féminin et $40 \%$ de sexe masculin. Ces personnes, dont le lieu de résidence est semi-urbain et rural, viennent recevoir des traitements divers et subir des examens cliniques. Ils sont ambulants et doivent faire preuve d'autonomie, pour bénéficier des services de cette hôtellerie.

Cette hôtellerie se divise en deux espaces bien distincts : le lieu de résidence et le lieu de traitements. Ceci reflète également la séparation physique de l'univers des soignés et de celui des soignants. Les espaces réservés à la résidence permettent aux personnes de s'isoler dans leur chambre ou de se regrouper, comme elles l'entendent. Les autorités favorisent que les temps libres soient réservés à la vie communautaire ; on cherche ainsi à éviter que tel patient ne souffre de trop d'isolement, risque toujours présent chez une personne qui percevrait son diagnostic comme une condamnation irréversible.

Notre insertion à l'hôtellerie a débuté en mars 1982. Deux techniques de recherche ont été privilégiées jusqu'à maintenant : l'observation participante et l'entrevue. Des entrevues ouvertes et semi-structurées ont été réalisées avec des soignés et des soignants et c'est surtout sur la base de ces matériaux qu'on a pu saisir les discours sur le cancer.

\subsection{Le cancer en tant que phénomène social}

\section{Retour à la table des matières}

Le cancer, comme on le sait, est la deuxième cause de mortalité dans la plupart des pays occidentaux, après les maladies cardiaques. On lui attribue $20 \%$ des mortalités. Le cancer du sein est la première cause de mortalité chez les femmes, alors que celui du poumon se révèle la deuxième cause de mortalité chez les hommes, au Canada. 
Le cancer est une maladie qui touche particulièrement les pays développés. Plusieurs organismes reliés à la santé ont affirmé, depuis au moins cinq ans, que le cancer est bel et bien une maladie dont les causes sont à rechercher dans notre environnement, et ce dans une proportion de 90\%. L'OMS et l'American Cancer Society sont de ceux-là. Malgré ce constat, la recherche mondiale se concentre surtout sur les aspects bio-médicaux, accordant une partie encore très minime des fonds à la recherche des causes environnementales. Depuis la célèbre déclaration du Congrès américain, en $1971^{12}$, les recherches se sont intensifiées. Malheureusement, l'incidence de la maladie demeure aussi élevée depuis 1950 et quelques formes de la maladie sont en croissance (tel que le cancer du poumon). Certains cancers sont mieux contrôlés que d'autres (la maladie de Hodgkin, par exemple), mais il n'en demeure pas moins que les cancers les plus fréquents (poumon, sein, côlon...) réservent à leurs porteurs des pronostics plutôt sombres. Lorsqu'on parle de guérison, en cancérologie, on ne doit pas oublier qu'on parle de survie à 5 ans pour un peu moins de la moitié de la population touchée. L'autre partie de la population meurt en deçà de 5 ans.

Bien qu'elle soit en réalité la seconde cause de mortalité dans les pays industrialisés, cette maladie est crainte plus que toute autre. Aiach (1980), qui a publié les résultats d'une enquête portant sur les facteurs culturels de différenciation en matière d'opinion et d'attitude vis-à-vis de la santé et de la maladie en France, s'est penché sur les représentations des maladies cardio-vasculaires et du cancer. Il a trouvé que l'image du cancer s'oppose symétriquement à celle des maladies cardio-vasculaires. Alors que ces deux maladies sont les plus redoutées, on croit généralement que le cancer tue davantage que les maladies cardio-vasculaires. On craint surtout son caractère mortel ; il semble effectivement profondément ancré dans la population comme l'image par excellence de la mort, à l'inverse des maladies cardio-vasculaires.

Par opposition au cancer, les maladies cardio-vasculaires font figure de maladies propres, nettes (s'opposant à l'idée de pourriture), qui ne traînent

12 Le Cancer Act fut autorisé par le Congrès américain en 1971, ce qui plaça la lutte contre le cancer au rang de priorité nationale. 
pas en longueur (...) et qui ne font pas souffrir. C'est, pour parler comme certains sujets interrogés, la maladie ou même la mort idéale (Aiach 1980 : 188).

Cette maladie engendre dans la population en général une série de fantasmes qui s'expriment de diverses manières, particulièrement dans les mass-média. Mystère et échec pour la médecine, elle s'offre également comme un phénomène social de premier ordre, par l'étendue et la diversité des mobilisations communautaires qu'elle suscite et par la puissance d'évocation des discours qu'elle provoque.

Le discours sur le cancer se présente assez spontanément comme un discours sur la mort. Dans la population, on reste toujours assez surpris de rencontrer une personne cancéreuse " qui ne semble pas malade ». Il arrive d'ailleurs assez souvent qu'inconsciemment, l'entourage s'adresse à cette dernière à l'imparfait (quand tu étais... lorsque tu faisais...) : la personne cancéreuse serait déjà d'un autre monde.

En est-il ainsi dans les milieux où la population des personnes cancéreuses constitue une majorité ? Entre eux, en tant que soignés, et entre les soignants, peut-on observer la récurrence de ce phénomène ? Est-ce que le discours sur le cancer dans la population en général peut se comparer à celui que partage une population atteinte de cette maladie et vivant en communauté ? Sans prétendre répondre à toutes ces questions, ces dernières nous motivent tout au moins au développement de la section suivante.

\title{
3. Le discours sur le cancer
}

\author{
3.1. Le discours des soignants
}

$\underline{\text { Retour à la table des matières }}$

À la suite des critiques nombreuses adressées à l'ordre médical concernant l'évitement de toute communication sur la vérité de la maladie avec le patient, nos institutions ont emboîté le pas et modifié 
quelque peu leurs attitudes face à la pression de l'opinion publique. La création d'un pavillon aux abords agréables, modernes, répond de plus en plus aux besoins d'humanisation des soins aux personnes cancéreuses, autrefois stigmatisées et isolées ${ }^{13}$. On cherche aujourd'hui à dédramatiser l'expérience du cancer : avant les années soixante-dix, soit avant le Cancer Act, la personne atteinte de cancer était le plus souvent cachée, victime du silence presque concerté de l'entourage, plus ou moins dépossédée de son humanité, reléguée à l'espace privé de la maison familiale en raison de l'impuissance des thérapeutiques médicales, ou quasi isolée dans l'espace clos de la chambre d'hôpital.

D'une maladie presque honteuse, encore confusément associée à la tuberculose, donc à la pauvreté et à la saleté, le cancer est devenu « une maladie des temps modernes », une " maladie de civilisation » et une cible de l'activité scientifique. Être porteur de cancer signifie plus ou moins devenir digne d'intérêt scientifique, objet de médiation entre le monde inaccessible de la haute technologie et le monde anonyme de la vie quotidienne.

Pour le médecin, d'ailleurs, le contact avec les patients cancéreux se révélait autrefois (soit il y a une quinzaine d'années) décevant et moins valorisant, puisque ces derniers mouraient presque tous. Aujourd'hui, les « succès » thérapeutiques obtenus et la haute estime dont jouissent les chercheurs et praticiens à l'œuvre dans la tâche d'éradication de ce «mal du siècle » rendent beaucoup plus désirable le statut de médecin-oncologue et diminuent à prime abord les difficultés de communication qu'impliquait autrefois la relation médecin/patient : le médecin et son équipe sont munis d'une mission spéciale.

Le statut de la personne cancéreuse s'est modifié. Dans cette hôtellerie, pourtant, malgré l'entreprise de dédramatisation à l'oeuvre dans ce milieu, on observe que les résidents ne connaissent pas toujours la nature de leur diagnostic. Le statut de cancéreux demeure ambigu. Un soignant a d'ailleurs souligné que le patient de l'hôtellerie, comparé au

13 Notons cependant que le milieu dans lequel sont recueillies les données n'avait pas pour premier objectif celui de l'humanisation des soins, mais répondait plutôt à des contraintes d'ordre économique. Il serait ici trop fastidieux de documenter en détail cette question, qui déborde le cadre de notre présentation. 
patient de l'hôpital, vit une situation où il n'apprend très souvent la nature réelle de sa maladie qu'à son entrée au Pavillon (si, bien sûr, il lui est donné de l'apprendre). Tout se passe comme si, dans les hôpitaux régionaux où les patients sont habituellement diagnostiqués, le personnel renvoyait parfois la responsabilité de l'annonce du diagnostic (et de ses conséquences) au personnel spécialisé du Pavillon. Il s'ensuit que le séjour à l'hôtellerie peut facilement être vécu comme un abandon du médecin traitant (de l'hôpital régional).

On tend toutefois à demeurer le plus ouvert possible à l'endroit du patient. Comme le mentionnait une infirmière en place dans ce centre depuis 25 ans : " Il y a 20 ans, les médecins n'osaient pas dire le mot cancer. On encourageait de façon désespérée et on mentait. Aujourd'hui, on nuance beaucoup plus ce qu'on dit, et on fonctionne de manière beaucoup plus individualisée. On écoute peut-être un peu plus. »

Malgré cet idéal d'ouverture à la communication, il n'en demeure pas moins que face à un patient qui ne connaît pas son diagnostic, on tendra à attendre les questions plutôt qu'à informer directement le patient. Il arrive ainsi qu'au bout d'un mois de séjour dans un Centre de traitement pour le cancer, un patient s'interroge encore « s'il a ou non le cancer » ou si sa maladie, "c'est bien le cancer ». Doit-on interpréter ce phénomène uniquement en terme de résistance du sujet face à la fatalité éventuelle du pronostic, ou ne peut-on pas questionner un certain mode de contrôle de l'institution quant aux informations qu'elle détient (ou ne détient pas) sur la maladie ? À quel moment parle-t-on de déni, quand thérapeute et client partagent des informations distinctes, selon l'univers sémantique et culturel qui marque leur dire ?

De fait, on craint encore fortement la responsabilité de l'annonce du diagnostic. Bien que certaines recherches mentionnent que le patient atteint d'un cancer préfère connaître la nature de son diagnostic, et ce dans $90 \%$ des cas, il semble que cela soit insuffisant pour motiver les cliniciens à adopter une attitude vraiment claire avec le patient (Weisman 1980).

Il existe un moyen pour le clinicien qui permet d'éviter l'effet de choc du diagnostic : il s'agit des stratégies de nomination du cancer. On parlera de « tumeur maligne », sans prononcer le vocable fatidique 
de cancer, qui semble répondre à l'intention de dédramatisation de la maladie. Cette appellation a toutefois le défaut de garder le patient dans une certaine forme d'ignorance, puisqu'il n'est jamais certain pour beaucoup d'entre eux qu'il s'agisse réellement de cancer. On se demandera, par exemple, si cancer et tumeur maligne sont équivalents : certains croient que oui et d'autres, non.

Plusieurs glissements sémantiques ont été d'ailleurs observés dans ce milieu, afin de nommer autrement certains éléments autrefois familiers à l'univers du patient cancéreux et de ses soignants. On a remplacé, ainsi, le terme d'anus artificiel ou anus contre nature par stomie, celui de cancer par tumeur, celui de cancérologie par oncologie. On a cherché ainsi à éviter toute connotation mortifère ou dramatique par l'emploi de termes, oserions-nous dire, plus aseptiques, c'est-à-dire médicaux, et dont la charge affective sera de moindre portée. Le mot cancer (très polysémique) évoquant la mort, la tristesse, l'abandon, etc... sera davantage imprégné de danger et de pouvoir symbolique que celui de tumeur, plus technique et moins connoté, qui lui sera préféré en raison de sa puissance d'évocation plus faible. Le mot cancer nous réfère à un état général de la personne, celui de tumeur nous décrit plutôt une excroissance localisée, identifiée à un endroit du corps.

Le cancer se révèle effectivement un symbole de mort dans la culture des soignants. Ce symbole, tout comme la maladie, devient une cible à exterminer dont on doit réduire l'étendue pour mieux en viser les effets. Les modifications introduites dans la terminologie des soignants en seraient des indices. Ce phénomène reflète également assez bien la pratique des soignants qui est, face au patient, de tendre à réduire l'expérience de la maladie à une affaire technique, de fait, à sa manifestation somatique. Toute l'attention accordée au patient, de la part des membres de l'équipe médicale, sera une attention portée à la tumeur et non pas au cancer.

Signalons également un phénomène qui soutient davantage ce que nous avançons. À chaque fois que nous avons eu à situer notre présence en tant qu'anthropologue dans ce milieu, et à expliciter davantage les buts du travail de recherche, on interprétait le sens de nos préoccupations, centrées, entre autres, sur la qualité de la vie, comme une recherche " à la Kübler-Ross », c'est-à-dire orientée vers l'approche 
aux mourants. Rappelons que la clientèle de l'hôtellerie reçoit majoritairement des patients dans une phase curative de la maladie, plutôt que palliative. Est-ce à dire que se préoccuper de qualité de vie équivaut nécessairement à parler de qualité du mourir ? Est-ce que l'expérience du cancer se réduit à une expérience tournée vers la mort ? Ou est-ce simplement que la personne cancéreuse, assujettie ou objectivée, ne possède plus en contexte clinique le pouvoir et l'autorité d'un être humain à part entière ?

Rappelons que les formes de cancer que l'on retrouve le plus fréquemment dans cette population sont parmi celles dont les succès thérapeutiques demeurent mitigés.

Ainsi, l'espérance de guérison à 5 ans pour un cancer du côlon serait à $39 \%$ chez les hommes et à $29 \%$ chez les femmes; on l'établit à $6,8 \%$ pour un cancer du poumon, pour les deux sexes, et à $44 \%$ pour un cancer du sein chez les femmes (Cattan et Mathé 1976) ${ }^{14}$.

Ceci pourrait expliquer l'attitude de certains médecins qui, sans mentir ouvertement à leur clientèle, usent de stratégies de discours qui permettent de taire ou de nuancer certaines informations, tout en évitant d'affirmer l'impuissance relative de leur profession vis-à-vis de cette maladie. On craint aussi, sans trop l'affirmer et probablement avec raison, l'effet morbide que pourraient avoir de telles déclarations sur l'évolution de la maladie du patient. Ceci contredit de manière flagrante les conceptions bio-médicales du cancer et démontre jusqu'à un certain point qu'on avoue l'influence d'autres ordres de phénomènes, sinon quant à l'étiologie, au moins sur le pronostic.

On pourrait résumer les propos qui précèdent en affirmant que dans le discours des soignants, le discours sur le cancer porte les marques d'une motivation à la dédramatisation, d'une part, mais aussi celles de stratégies qui permettent d'éviter la confrontation à la position d'impuissance et ce, malgré un encouragement à l'ouverture à la communication franche dans la relation thérapeutique. Ceci permet probablement la maintenance et la reproduction de conditions de communi-

14 Les statistiques les plus récentes sont disponibles dans $C a A$ Cancer journal for clinicians, Vol. 33, no 1. 
cation supportable entre les soignés et les soignants, mais rend aussi possibles des phases curatives de plus en plus longues, au coût d'une qualité de vie parfois douteuse. On pourrait ajouter à ces propos que le discours sur le cancer apparaît dans le monde des soignants, notamment celui des médecins, comme un discours sur la mort en tant qu'objet d'échec.

\subsection{Le discours des soignés}

$\underline{\text { Retour à la table des matières }}$

Pour les soignés, l'hôtellerie est habituellement décrite comme un lieu agréable, bien qu'on s'y ennuie beaucoup. La personne ne connaît pas l'heure de son traitement et est appelée (interpellée ?) à un moment ou l'autre de la journée, par un système d'intercom. Le temps nécessaire au traitement est relativement court (5-15 minutes). Certains soignés ont employé la figure de la gare pour décrire la situation d'attente et de passivité dans laquelle ils sont placés.

Entre les personnes cancéreuses, on observe également une certaine crainte à utiliser le mot cancer. Certains l'utiliseront en riant (dans les groupes d'hommes, par exemple), un peu comme pour en braver les effets potentiels. Il est vrai qu'on cherche aussi à observer d'une certaine manière le règlement de l'hôtellerie (d'ailleurs plus une incitation qu'un règlement) invitant les gens à ne pas parler de « la maladie » et « à laisser la carte d'assurance-maladie dans leur poche ». On cherche ainsi à ne pas faire de l'hôtellerie un lieu trop morbide, et ainsi à dédramatiser (là encore) l'expérience de la maladie. On évite ainsi, par un certain contrôle, une charge affective qu'on juge lourde et trop complexe à supporter.

Par ailleurs, certains patients avouent ouvertement leur « peur du mot » : ils en confirment ainsi en partie ses connotations mortifères. Les patients s'entendent assez peu sur l'endroit et les circonstances où on peut effectivement en parler, et produisent des réponses très variables concernant la fréquence d'évocation de la maladie dans le milieu. Il semble que cette réponse dépende en grande partie du type de rela- 
tion que le patient entretient avec sa propre maladie, selon qu'elle provoque plus ou moins d'angoisse.

Chez les soignés, une certaine terminologie empruntée quelque peu au discours savant module la perception de l'intensité de la maladie chez soi et chez l'autre. Un nodule est un cancer négligeable et le kyste lui est équivalent ; un ganglion devient assez sérieux ; il peut devenir cancéreux, S'il n'est pas enlevé par chirurgie ou radiothérapie ; une tumeur est parfois cancéreuse, parfois non, mais dans la majorité des cas, sera diagnostiquée comme moins sévère qu'un cancer lui-même. Seul le substantif cancer fait consensus chez les soignés pour identifier précisément la maladie.

On a souvent interprété l'attitude peu loquace des personnes cancereuses au sujet de leur maladie comme un déni de leur réalité. Ils affirment plutôt craindre de « fatiguer les autres avec leurs affaires » (la maladie est le lieu d'une individualité, d'une intimité) et ne pas vouloir comparer leur état avec celui des autres (qui pourrait être pire et faire peur au voisin, ou être moins pire, alors que celui du voisin serait pire...). On craint ici que l'évocation directe de la maladie n'influence l'évocation du mal, chez soi ou chez l'autre.

Bien que la peur de contagion soit assez peu commune (sauf chez les personnes âgées), on remarque que les gens se touchent très peu entre eux. On maintient généralement une attitude de discrétion, très appréciée, concernant la révélation de son état et le discours sur sa maladie ; on préfère aussi ne pas toucher, puisque même si on "sait » que la maladie n'est pas contagieuse, un doute demeure, car on ne connaît pas véritablement la cause exacte de la maladie. Il est par ailleurs difficile d'affirmer que le cancer soit l'unique déterminant de ce comportement, compte tenu qu'en soi le toucher représente un mode d'expression très limité dans notre culture.

Certains patients affirmeront que, d'ailleurs, ce n'est plus de « maladie » que l'on parle, mais de « traitement » : en attente de guérison, on est donc plus tout à fait malade. Parler du traitement (son effet, ses propriétés, etc.) semble moins difficile que de parler de sa maladie directement. Cette tactique permet aussi de réduire l'expérience de la maladie à sa seule composante biologique, conformément au discours 
des soignants, et d'extirper de la maladie son intense pouvoir symbolique (et mortifère).

Bien que le processus de dédramatisation de l'expérience de la maladie soit à l'œuvre tant dans le discours des soignés que dans celui des soignants, d'autres éléments du discours des soignés permettent d'affirmer que ces derniers connectent au symbole du cancer d'autres caractères, ceux-là moins strictement reliés à la mort qu'à la notion de modernité. Les commentaires qui suivent tentent d'en faire la démonstration.

La plupart des informateurs s'entendent à dire que le cancer est un nouveau mot plutôt qu'une nouvelle maladie. Les propos suivants illustrent assez bien cette tendance :

Le cancer... ça a toujours existé... Avant, les gens mouraient on ne savait pas de quoi... de consomption... de coliques... ils mouraient ... aujourd'hui on a donné un nom à ça, ils ont trouvé le nom de la maladie ... mais on sait que ça a toujours existé.

On s'entend toutefois à dire qu'il y a plus de cancers aujourd'hui qu'autrefois ; plus de cancers, mais aussi plus de maladies de toutes sortes. On se demande si cela est dû au fait du perfectionnement de la médecine, laquelle identifie davantage de maladies (leur donne un nom) et les détecte aussi plus tôt.

L'autre hypothèse sur laquelle on insiste le plus considère l'effet de l'état de saleté chronique de l'environnement québécois (et du monde moderne en général) sur la santé. En effet, si on affirme que peut-être il y aurait plus de cancers, on invoque souvent la multiplication des produits chimiques, le peu d'attention à l'environnement naturel, la pollution atmosphérique ou les centrales nucléaires, pour expliquer cette maladie « de civilisation ». Sontag (1978) affirmait que le discours sur le cancer est marqué des signes de la surproduction ; nous ajouterions qu'il est également, du moins chez les soignés que nous avons rencontrés, marqué des signes de la modernité. Les changements jugés trop rapides, le stress (ici équivalent à rythme de vie), voire l'absurdité dans laquelle la planète semble s'engouffrer se connectent et donnent forme au symbole du cancer. 
Exprimé différemment, la modernité produit notre propre mort et nous en sommes les victimes. Les propos suivants tenus par des patients, le soutiennent :

Le cancer ça... c'est un mot récent... avant, y parlaient pas de ça... je me rappelle quand j'étais jeune... personne ne parlait de ça, la majorité vivait tous assez vieux... on meurt plus jeune aujourd'hui... je le sais pas... ça devait exister avant, mais je le sais pas... certainement... c'était moins pollué qu'aujourd'hui... y avait moins de changement qu'aujourd'hui... c'était moins pollué... c'est pour ça que ça marche pas... comme tous les barrages qui ont fait là, c'est pas normal... les pluies qu'on a là, c'est pas normal... la température qu'on a là, c'est pas normal... tout s'en suit... tous les essais qu'y font... dans la mer et dans l'eau... c'est par là qu'on va tous mourir... j'sais pas si je me trompe... mais moi je prends ça de même...

Ça doit être la pollution dehors... tout ce qui se déverse dans les eaux... les acides... les caustiques... si tu verrais ça à la " choppe »... ce qu'ils envoient à l'eau... y a des tests à passer il les font pas... y répandent leurs cochonneries... les poissons, le diable l'emporte... pis l'eau qu'on boit... pour moi, les maladies ça vient de là ... va falloir qu'on fasse quelque chose... tout est cancer... du caustique ... du chlore... de l'acide... des fois, c'est tellement fort que les tuyaux percent... nous autres on respire ça... on est chanceux d'avoir de bonnes tripes...

Le discours des soignés, s'il est entre autres un discours détourné sur la mort, ou sur le cancer symbole de mort, apparait aussi comme un discours sur la modernité porteuse de mort. Son objet, plutôt que de tendre à se refermer sur ses référents somatiques, cherche au contraire à s'ouvrir sur les connotations aux colorations nettement culturelles, où le sujet de l'énonciation trouve sens à son existence au moyen d'une construction de l'esprit qui fait aussi de sa mort celle de la mort d'un monde, de celui qui l'a rendu vivant. Il rejoint, curieusement, le discours de certains spécialistes qui manipulent le discours sur la mort et le pervertissent, faisant de ce dernier le discours sur la fin d'un monde. Dans notre cas, la maladie sert de prétexte à l'élaboration d'un discours, lequel use d'une situation subjective, contextuelle, 
afin de réfléchir sur l'évolution du monde et sur son avenir. Et ceci à un tel point qu'il est parfois difficile de saisir de quelle mort on parle... 


\section{Conclusion}

$\underline{\text { Retour à la table des matières }}$

La présentation qui précède nous aura informé principalement de deux propositions. La première serait que le discours sur le cancer pourrait être l'une des formes possibles qu'emprunte le détournement du discours sur la mort dans la formation sociale québécoise. La deuxième serait que le discours des personnes atteintes du cancer, en plus d'être une métaphore du discours sur la mort, se montre aussi comme un discours sur la modernité porteuse de mort. Il rejoint en ce sens la réflexion des chercheurs les plus érudits en prétextant un discours sur le cancer, et sur la mort, pour réfléchir aussi sur les conditions concrètes de la modernité.

Au cours de cette présentation, nous avons peu abordé la question du déplacement du discours religieux sur le discours médical. Nous considérions simplement que, sur le plan empirique, les résultats, très partiels répétons-le, d'une recherche ethnographique dans un milieu d'oncologie clinique en seraient une illustration possible.

Nous devrions nous permettre ici une dernière remarque. Nous avons particulièrement insisté sur les connotations mortifères du symbole du cancer, tant dans l'univers symbolique des soignés que dans celui des soignants. On ne saurait cependant réduire l'expérience de la personne cancéreuse à ces aspects de son activité symbolique : ceci fausserait nos interprétations et celles que nous donnent à entendre les personnes cancéreuses. L'organisation du monde clinique occulte l'expérience du patient et permet d'interpréter la demande de guérison du Patient, dans le sens strict d'une demande de conservation de la vie à tout prix. Cette dimension, certes, est présente dans ta demande du soigné au soignant, mais on doit s'interroger sur le fait qu'il semble qu'on ne s'attache généralement qu'à chercher à répondre à cette seule partie de la demande de guérison. Il appert que cette réponse marque bien les préoccupations des soignants, liés professionnellement aux 
fonctions instrumentales de leur pratique, déléguant à d'autres et pour plus tard (à la phase terminale, par exemple) les fonctions expressives. Une personne cancéreuse peut vivre plusieurs années avant d'atteindre la phase terminale éventuelle. Cette phase de la vie, chez les soignés, leur impose, outre des préoccupations quant à leur devenir et leur finalité, d'autres préoccupations, plus pragmatiques, touchant à l'organisation quotidienne de l'existence qui sera plus ou moins réduite. Ceci, bien que nous n'ayons pas abordé cette question, fait également partie de la demande de guérison et c'est justement ces aspects de la demande qui tendent à être occultés dans le monde clinique. La réduction de l'expérience du cancer à une expérience tournée vers la mort pourrait être, dans le monde clinique du moins, davantage caractéristique de l'univers des soignants que de celle des soignés.

\section{RÉFÉRENCES}

$\underline{\text { Retour à la table des matières }}$

AIACH, P., Peurs et images de la maladie : l'opposition cancer/maladies cardiaques. Bulletin du cancer, vol. 67, no 2, 1980, pp. 183-198.

ARIÈS, P., Essai sur l'histoire de la mort en Occident du MoyenÂge à nos jours. Paris, Seuil, 1975.

BAUDRILLARD, J., L'échange symbolique et la mort, Paris, Gallimard, 1978, coll. Bibliothèque des sciences humaines.

CHAMBOREDON, J.C., La restauration de la mort, objet scientifique et phantasmes sociaux. Actes de la recherche en sciences sociales. vol. 2, no 3, 1976, pp. 78-87.

GLASER, R. and STRAUSS, A., Awarness of dying -Chicago, Aldine Publishing Co., 1965.

GORER, G., The pornography of death. Encounter, 1955. 
HERTZ, R., Contribution à une étude sur la représentation collective de la mort. Sociologie religieuse et folklore, Paris, PUF., 1970

HERZLICH, C., Le travail de la mort. Annales. 31 : 1, pp. 197218.

JAULIN, R., La mort sara. Paris, Plon, 1967.

KÜBLER-ROSS, E., On death and dying. New York, MacMillan Pub. Co, 1970.

LASCH, C., The culture of narcissism. New York, Warner Books, 1979.

LEVINSON, D., An overview of the euthanasia movement in the U.S. Today. Essence, vol. 3, no 1, 1979, pp. 21-31.

MATHE, G. et CATTAN, A., Cancérologie. Paris, Expansion scientifique française, 1976.

MATARASSO, M., Thanatos : Crise et critique. Cahiers internationaux de sociologie, LXII, 1977, pp. 89-104.

RACINE, L., Nouvelles thérapies et nouvelle culture. Sociologie et sociétés. vol. 9, no 2, 1977, pp. 34-55.

SAILLANT, Francine , Le cancer comme symbole de mort. (Note de recherche). Anthropologie et sociétés, vol. 6, 3, 1982, pp. 91-103.

, Mourir : un processus qui débute avant la phase terminale. Santé mentale au Québec, vol. 8, 5, juin 1973.

SONTAG, Susan, Illness as metaphor. New York. Farrar, Strauss and Giroux, 1978.

THOMAS, L.-V. 
, Anthropologie de la mort. Paris, Payot, 1976. , Mort et pouvoir. Paris, Petite bibliothèque Payot, 1978.

VALADE, B., L'idée de décadence. Cahiers internationaux de sociologie. LXII, 1978, pp. 75-88.

WEISMAN, A.D., Coping with cancer. New York, McGraw-Hill, 1979.

ZIEGLER, J., Les vivants et la mort. Paris, Seuil, 1975, coll. Points.

Fin du texte 